\title{
The Spatiotemporal Oscillations of Order Parameter for Isothermal Model of the Surface-Directed Spinodal Decomposition in Bounded Binary Mixtures
}

\author{
Igor B. Krasnyuk ${ }^{1}$ and Roman M. Taranets ${ }^{2}$ \\ ${ }^{1}$ A. A. Galkin Donetsk Institute for Physics and Engineering, National Academy of Sciences of Ukraine (NASU), \\ 72, R. Luxemburg Street, 83114 Donetsk, Ukraine \\ ${ }^{2}$ Institute of Applied Mathematics and Mechanics, National Academy of Sciences of Ukraine (NASU), \\ 74, R. Luxemburg Street, 83114 Donetsk, Ukraine \\ Correspondence should be addressed to Roman M. Taranets, taranets_r@yahoo.com \\ Received 30 September 2008; Accepted 29 December 2008 \\ Recommended by Peter McClintock
}

The asymptotical behavior of order parameter in confined binary mixture is considered in one-dimensional geometry. The interaction between bulk and surface forces in the mixture is investigated. Its established conditions are when the bulk spinodal decomposition may be ignored and when the main role in the process of formation of the oscillating asymptotic periodic spatiotemporal structures plays the surface-directed spinodal decomposition which is modelled by nonlinear dynamical boundary conditions.

Copyright (C) 2009 I. B. Krasnyuk and R. M. Taranets. This is an open access article distributed under the Creative Commons Attribution License, which permits unrestricted use, distribution, and reproduction in any medium, provided the original work is properly cited.

\section{Introduction}

The problem of self-organization for order parameter in binary mixtures, which are confined with two flat walls, is important in physics of condense matter $[1,2]$. At first, for the binary mixtures, this problem was considered by Binder, Puri, and Frisch. The existence of oscillating distributions of the order parameter (on a space variable for each fixed moment time) has been proved $[2,3]$. Binder and Frisch considered also the Cahn-Hilliard $(\mathrm{CH})$ equation for conserved-order parameter with nonlinear dynamical boundary conditions [1] and described a phase diagram for the problem. However, it is unknown what is being an attractor of the considered dynamical binary system, that is, what is being an asymptotic behavior of the solutions of such imposed boundary-value problem as a time $t \rightarrow \infty$.

The aim of the present paper is to establish how the attractor of trajectories for this problem is constructed. The achievements of the paper are as follows: (i) we show that the solutions tend to the limit functions as $t \rightarrow \infty$ with a finite set of discontinuities which are determined by topological properties of the functions $F_{k}, k=1,2$ in the boundary conditions (in this case, we have the solutions of relaxation non-Fickian diffusion type); (ii) there are limit solutions with infinite (countable) and infinite (uncountable) number of discontinuities (in such case, we have the solutions of preturbulent type and turbulent type; accordingly, the set of points of discontinuities for the solutions of turbulent type is familiar to a Cantor-like set); (iii) there exist the solutions whose limit functions are discontinuities at all points. This solutions take up a particular place among the solutions and they lead to the appearance of the so-called "deterministic randomness," namely, in order to characterize the long-time behavior of the solutions, it is necessary to proceed to a probabilistic description [4].

In such way, we consider the problem which was introduced in [1], but instead of the classical $(\mathrm{CH})$ equation, we research the so-called modified Cahn-Hilliard $(\mathrm{mCH})$ equation which is proposed by Galenko et al. $[5,6]$. The modified equation is the model for diffusion and phase separation which takes into account hyperbolic relaxation of 


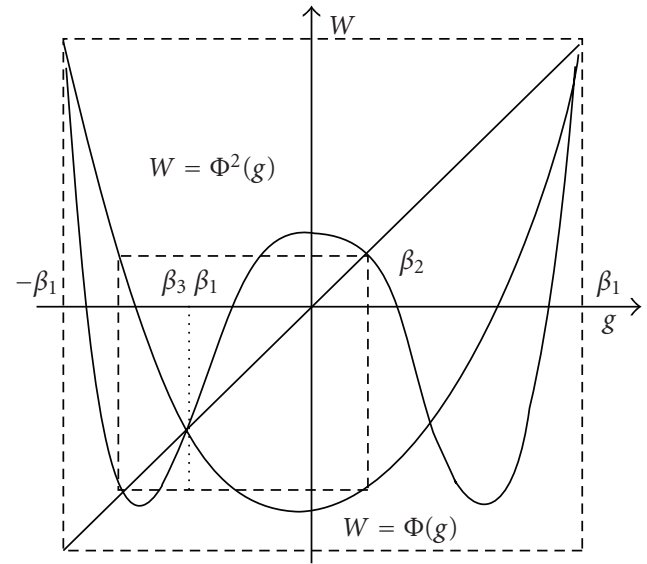

(a)

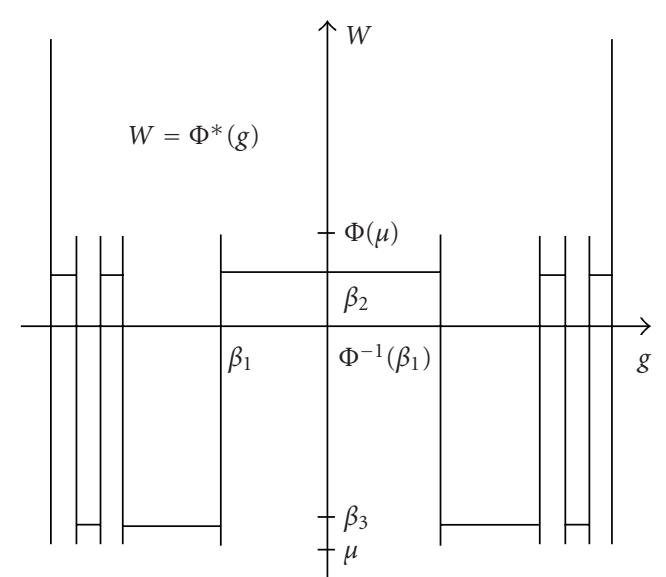

(b)

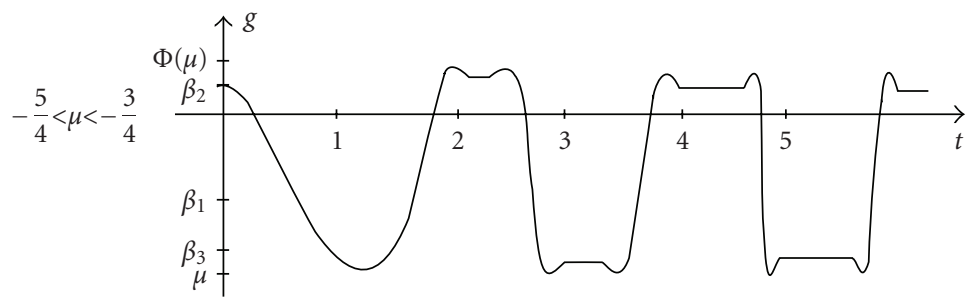

(c)

FIgURE 1: Asymptotically periodic distributions of the order parameter of the relaxation type. The map $\Phi: g \mapsto g^{2}+\mu$. Here, $\beta_{1}$ and $\beta_{3}$ are stable ordered phases, and $\beta_{2}$ is unstable disordered phase. If the amplitude of initial bubbles is greater than $\beta_{2}$, then there are the oscillations. If the amplitude of initial bubbles is less than $\beta_{2}$, then asymptotic distributions of order parameter tend to the constant value $\beta_{1}$ [4].

the solute diffusion flux $I$. The flux can be determined by the formula (see $[5,6])$

$$
\begin{aligned}
I\left(\cdot, t+\tau_{D}\right) & =I(\cdot, t)+\tau_{D} I_{t}(\cdot, t)+O\left(\tau_{D}\right) \\
& =-M(u)\left(\mathcal{F}_{u}^{\prime}(u)-\epsilon_{u}^{2} u_{x x}\right)_{x}(\cdot, t),
\end{aligned}
$$

where $O\left(\tau_{D}\right) \rightarrow 0$ as $\tau_{D} \rightarrow 0, u$ is the order parameter, $\tau_{D}$ is the delay time, $M$ is the atomic mobility, $\mathcal{F}_{u}^{\prime}$ is the $u$-derivative of the free energy $\mathcal{F}$. We denote that $\epsilon_{u}^{2}=$ $2 F_{0} \zeta_{b}^{2}$, where $F_{0}$ is connection energy [6] which is normalized by $k_{B} T$. Here, $k_{B}$ is the Boltzmann constant, and $\zeta_{b}$ is the bulk correlation length. A few advancements were made for strongly nonequilibrium phase separation. In 1986, Galenko and Lebedev [6] generalized the linearized $(\mathrm{CH})$ equation to the case of existence of slowly relaxing variable. Their calculations show that the instability of the system is not standard diffusive type but rather it is controlled by the relaxation of a slow structural variable such as the diffusion flux determined by the formula (1).

In this paper, we consider the case only when the order parameter varies slowly in the bulk, and we determine the condition when this supposition is true. In this case, asymptotically stable equilibrium states of the system may be stabilized by nonlinear boundary conditions which are described by surface-directed spinodal decomposition (SDSD) in the binary system. As a result, the SDSD determines the limit distributions of order parameter which have the form of traveling waves and its graphes are asymptotic periodic functions with finite, infinite (countable), or infinite (uncountable) number of discontinuities (Figure 1).

\section{Formulation of the Problem}

We consider binary mixtures with diffusion flux (1) and, additionally, we suppose that the equation $u_{t}+I_{x}=0$ is true. Then, from (1), we obtain the $(\mathrm{mCH})$ equation:

$$
\tau_{D} u_{t t}+u_{t}=\left[M(u)\left(\mathcal{F}_{u}^{\prime}(u)-\epsilon_{u}^{2} u_{x x}\right)_{x}\right]_{x} .
$$

In [6], it is shown that (2) takes place for bulk spinodal decompositions with locally nonequilibrium diffusion. In what follows, we consider the boundary conditions in the common form [1]:

$$
\left.u_{t}\right|_{x=x_{k}}=\left.F_{k}\left(h_{k}, g_{k}, v, u, u_{x}, u_{x x}, u_{x x x}\right)\right|_{x=x_{k}}
$$

for the solutions of (2). Here, $x_{1}=0, x_{2}=l$ and $F_{k}$ are given functions, $h_{k}$ describes surface attractive potentials (for magnetic liquid, it may be surface magnetic field [1]), and $g_{k}=J_{s}^{(k)} / J^{(k)}$, where $J^{(\cdot)}$ is modelled the bulk forces and $J_{s}^{(\cdot)}$ is modelled surface forces (in detail see $[1,2]$ ). Next, $v$ is a constant (or a function) which is proportional to the bulk correlation length $\zeta_{b}$. In [1], for example, it was considered (3) for $F_{k}=h_{k}+g_{k}(u)+v u_{x}-(v / 4)^{2 / 3} u_{x x}-(v / 4)^{1 / 3} u_{x x x}$. In the simplest case, if $F_{1}=h_{1}+g_{1} u(0, t)$, then $u(0, t)=$ 
$u(0,0) \exp \left(g_{1} t\right)-h_{1} / g_{1}$. Thus, if $g_{1}>0$, then the surface order parameter increases in time, consequently, and the wall $x=0$ is attractive. If $g_{1}<0$, the wall $x=0$ is repulsive. In [2], it was considered (3) for $F_{k}=\left(\tau_{v} / \tau_{s}\right)\left(-u_{x}-h_{k} / v-(1 / v) g_{k}(u)\right)$, where $\tau_{s}$ and $\tau_{v}$ are the surface and bulk relaxation times accordingly. In typical cases $\tau_{s} \ll \tau_{v}[1]$.

Further, the self-organized structures (as in Figure 1) appear if and only if the Pfaffian form $F_{2} d u_{1}-F_{1} d u_{2}=$ 0 is integrable, that is, it can be reduced to the form $d W\left(u_{1}, u_{2}\right)=0$, where $W$ is the known function, and $u_{k}=$ $u\left(x_{k}, t\right)$. From the last equality, it follows that

$$
u(l, t)=\Phi_{\mu}(u(0, t)), \quad \mu=\mu\left(h_{k}, g_{k}, \nu\right),
$$

where $\Phi_{\mu}$ is some function. So, if the Pfaffian form is integrable, then (3) may be reduced to (4). Next, we consider the initial conditions $u(x, 0)=u^{(0)}(x), u_{t}(x, 0)=u^{(1)}(x)$. In common case, analytical solutions of this problem are unknown; but the boundary-value problem may be reduced to some simpler problem for spatial functions $F_{k}$ and constant mobility $M$. The problem has unique solution such as in Figure 1. We confined ourselves to the polymerpolymer systems with the components $A, B$ and the power of polymerization $N_{A}, N_{B}$, accordingly. The free energy (see $[5])$ is

$$
\mathscr{F}=\frac{u_{A}}{N_{A}} \ln u_{A}+\frac{u_{B}}{N_{B}} \ln u_{B}+\kappa u_{A} u_{B},
$$

where $u_{A}+u_{B}=1$, and $\kappa$ is Flory-Huggins parameter. We denote $u_{A}=u, u_{B}=1-u$, and we consider, for simplicity, the case $N_{A}=N_{B}=N$. Then, we find from (5) by the linear approximation that

$$
\widetilde{F}_{u}^{\prime}=\left(\frac{4}{N}-2 \kappa\right)\left(u-\frac{1}{2}\right)
$$

at a neighborhood of $u=1 / 2$. Then, perturbations $u \mapsto u-$ $1 / 2$ of the equilibrium state $u=1 / 2$ can be considered as the order parameter. As a result, due to (6), equation (2) can be rewritten in the form

$$
u_{t t}+\lambda u_{t}=D_{1} u_{x x}+D_{2} u_{x}^{2}-D_{3} u_{x x x x}-D_{4} u_{x} u_{x x x},
$$

where $t \mapsto t / \tau_{v}, x \mapsto x / l, \lambda=\tau_{v} / \tau_{D}$, and $D_{1}=$ $\left(M(1 / 2) \tau_{v}^{2} / \tau_{D} l^{2}\right)(4 / N-2 \kappa), D_{2}=\left(M_{u}^{\prime}(1 / 2) \tau_{v}^{2} / \tau_{D} l^{2}\right)(4 / N-$ $2 \kappa), D_{3}=M(1 / 2) \varepsilon_{u}^{2} \tau_{v}^{2} / \tau_{D} l^{4}, D_{4}=M_{u}^{\prime}(1 / 2) \varepsilon_{u}^{2} \tau_{v}^{2} / \tau_{D} l^{4}$. Here, $l$ is the spatial length, $\tau_{D}=M \alpha_{j}, \alpha_{J}$ is the coefficient specifying non-Fickian diffusion (see [6]). So, $\tau_{D}$ is the relaxation time of the diffusion flux to its steady state. Further, we suppose that $D_{2} \ll 1$ and $D_{4} \ll 1$, in particular, it is possible when $M(u) \sim u(1-u)$. Hence, we get from (7) that

$$
u_{t t}+\lambda u_{t}=D_{1} u_{x x}-D_{3} u_{x x x x} .
$$

Next, we consider the boundary conditions

$$
\frac{\tau_{s}}{\tau_{v}} u_{t}\left(x_{k}, t\right)=h_{k}+2 g_{k} u\left(x_{k}, t\right)
$$

for the solutions of (8), where $h_{k}$ and $g_{k}$ are some constant (in common case, they may be given functions). Moreover, we suppose that

$$
u_{x}(0, t)=0, \quad u_{x}(l, t)=0 .
$$

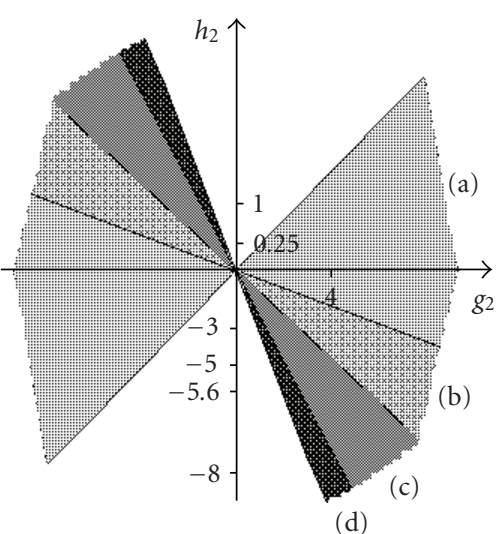

FIgURE 2: Phase diagram is obtained for the problem with nonlinear boundary conditions, which cause the surface-directed spinodal decomposition, by the theoretical method. Here (a) is the region of unique stable phase; (b) is the region of the spinodal decomposition; (c) is the region of the lamellar phase; (d) is the region of stochastic distributions of the order parameter.

\section{Reduction to the Difference Equation with Continuous Time}

Let

$$
u(x, t)=f(\zeta)+g(\eta),
$$

where $v=\sqrt{D_{1}}, \zeta=t+x / v, \eta=t-x / v$, and $f, g$ are arbitrary functions. Substituting (11) into (8), we deduce

$$
\frac{\beta}{v^{4}} f_{\zeta}^{\prime \prime \prime \prime}+f_{\zeta}^{\prime}=0, \quad \frac{\beta}{v^{4}} g_{\eta}^{\prime \prime \prime \prime}+g_{\eta}^{\prime}=0,
$$

where $\beta=M(1 / 2) \varepsilon_{u}^{2} \tau_{v} / l^{4}$. Integrating (12) along characteristics $d x / d t= \pm v$, we arrive at the differential-difference equation

$$
\frac{\beta}{v^{4}} Y^{\prime \prime \prime}(t)+Y(t)=0,
$$

where $Y(t)=f(t+l / v)-g(t-l / v)$. Here, we used the relations $f(t)=g(t)$ and $f^{\prime \prime \prime}(t)=g^{\prime \prime \prime}(t)$ accurate within a constant. In one's turn, these relations follow from the first condition of (10). The general solution of (13) is

$$
Y(t)=C_{1} e^{-2 v t}+C_{2} e^{\nu t} \cos (\sqrt{3} v t)+C_{3} e^{\nu t} \sin (\sqrt{3} v t),
$$

where $v=(1 / 2)\left(v^{4} / \beta\right)^{1 / 3}$. It follows from the definition of $Y(t)$ and functional relation (4) that

$$
f\left(t+\frac{l}{v}\right)=\Phi_{\mu}(f(t))+Y(t)
$$

where $f \mapsto 2 f$. Henceforth, we consider the case $Y(t) \simeq$ const, that is, $v t \ll 1$. Hence, we have two-parametric family of difference equations. The typical solutions of these equations are asymptotic periodic functions, such as in Figure 1. For example, let $F_{k}$ be from (9). Then, from the above procedure, we find the first integral

$$
c_{0}\left(h_{1}+2 g_{1} u(0, t)\right)^{g_{2} / g_{1}}=h_{2}+2 g_{2} u(l, t),
$$


where $c_{0}$ is a constant. Let $h_{1}=0, g_{2} / g_{1}=2$. These values correspond to experimental values from [2]. From (16), we find

$$
u(l, t)=\frac{c_{0} g_{2}}{2} u^{2}(0, t)-\frac{h_{2}}{2 g_{2}} .
$$

For the sake of simplicity, we choose $c_{0} g_{2} / 2=1$. Thus, it follows from (17) that

$$
f\left(t+\frac{l}{v}\right)+g\left(t-\frac{l}{v}\right)=(f(t)+g(t))^{2}+\hat{\mu}
$$

where $\hat{\mu}=-h_{2} / 2 g_{2}$. Next, we deduce from (10), that is, $f(t)=g(t)+c_{1}$ and $f(t+l / v)=g(t-l / v)+c_{2}$, that

$$
2 f\left(t+\frac{l}{v}\right)+c_{2}=\left(2 f(t)+c_{1}\right)^{2}+\hat{\mu}
$$

where $c_{1}=f(0)-g(0), c_{2}=f(l / v)-g(-l / v)$, and $f(0), f(l / v), g(0)$ and $g(-l / v)$ depend on initial functions $u^{(0)}$ and $u^{(1)}$. Let $z(t)=2 f(t)+c_{1}$. Then, it follows from (19) that

$$
z\left(t+\frac{l}{v}\right)=z^{2}(t)+\mu, \quad \mu=\hat{\mu}+c_{1}-c_{2}
$$

\section{Phase Diagram}

The phase diagram for the solutions of (20) is shown on Figure 2. This diagram is similar to corresponding diagram which was obtained by numeric simulation in $[1,2]$, where it was considered similar boundary problem to $(\mathrm{CH})$ equation [2].

\section{References}

[1] K. Binder and H. L. Frisch, "Dynamics of surface enrichment: a theory based on the Kawasaki spin-exchange model in the presence of a wall," Zeitschrift für Physik B Condensed Matter, vol. 84, no. 3, pp. 403-418, 1991.

[2] S. Puri and K. Binder, "Surface-directed spinodal decomposition in a thin-film geometry: a computer simulation," Journal of Statistical Physics, vol. 77, no. 1-2, pp. 145-172, 1994.

[3] I. B. Krasnyuk, "Oscillatory solutions to the system of AllenCahn and Cahn-Hilliard equations: a spinodal decomposition model," Physics of the Solid State, vol. 48, no. 11, pp. 2161-2170, 2006.

[4] A. N. Sharkovsky, Yu. L. Maistrenko, and E. Yu. Romanenko, Difference Equations and Their Applications, Mathematics and Its Applications, Kluwer Academic Publishers, Dordrecht, The Netherlands, 1993.

[5] P. Galenko and D. Jou, "Diffuse-interface model for rapid phase transformations in nonequilibrium systems," Physical Review E, vol. 71, no. 4, Article ID 046125, 13 pages, 2005.

[6] P. Galenko and V. Lebedev, "Analysis of the dispersion relation in spinodal decomposition of a binary system," Philosophical Magazine Letters, vol. 87, no. 11, pp. 821-827, 2007. 

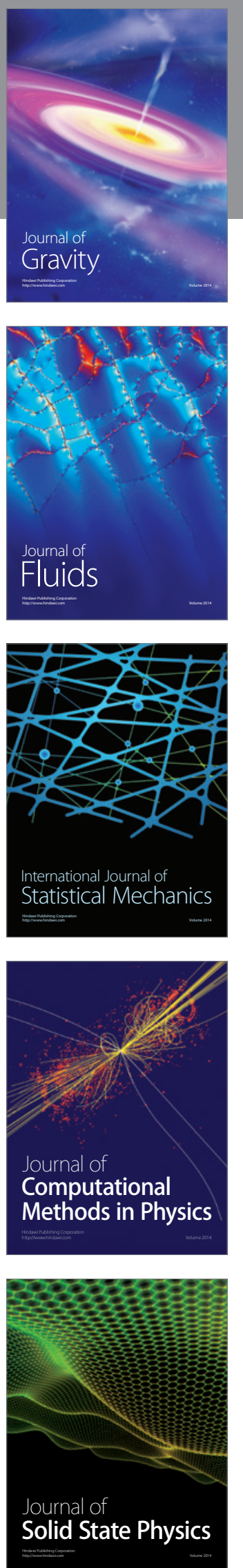

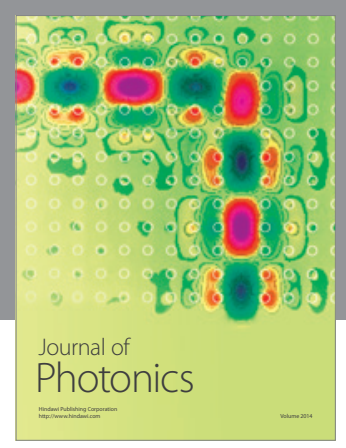

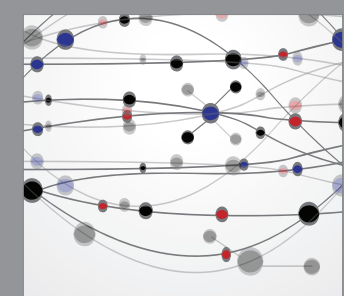

The Scientific World Journal
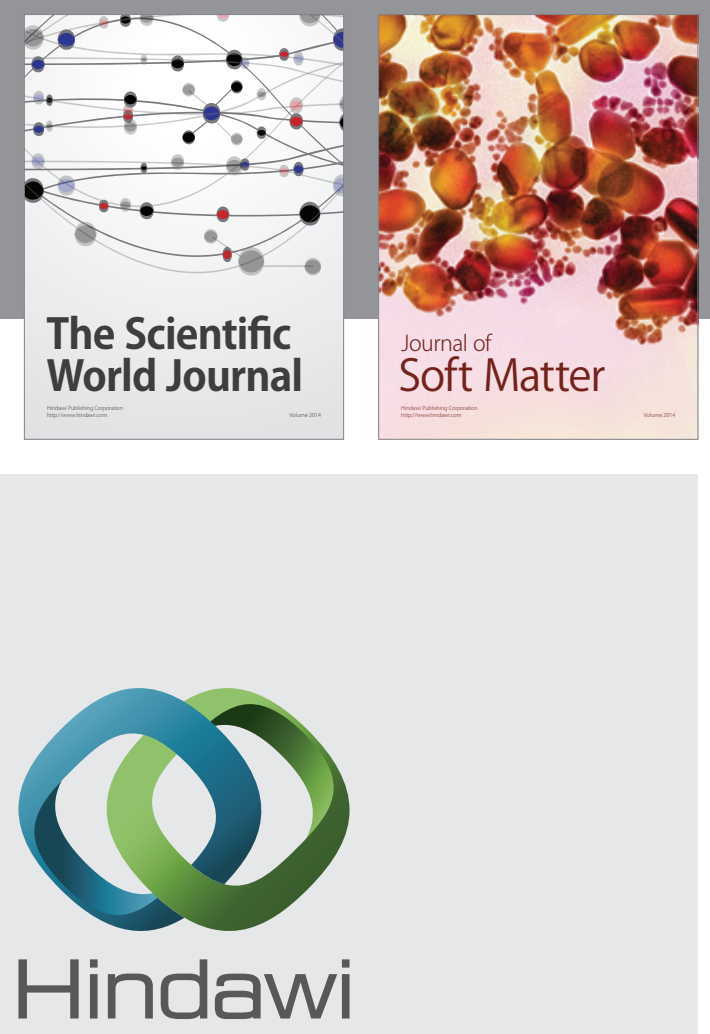

Submit your manuscripts at

http://www.hindawi.com
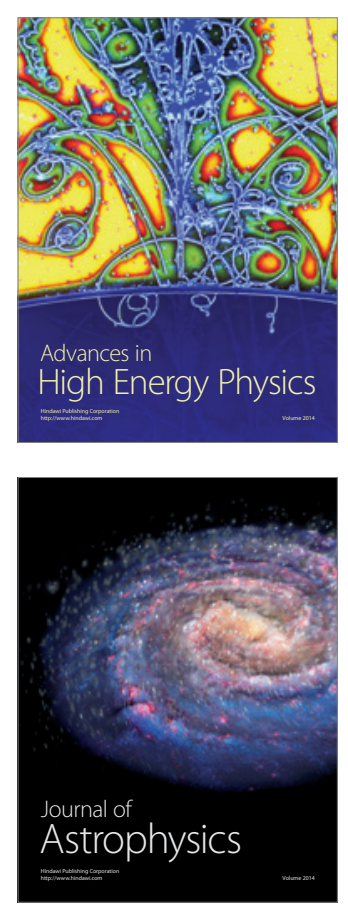
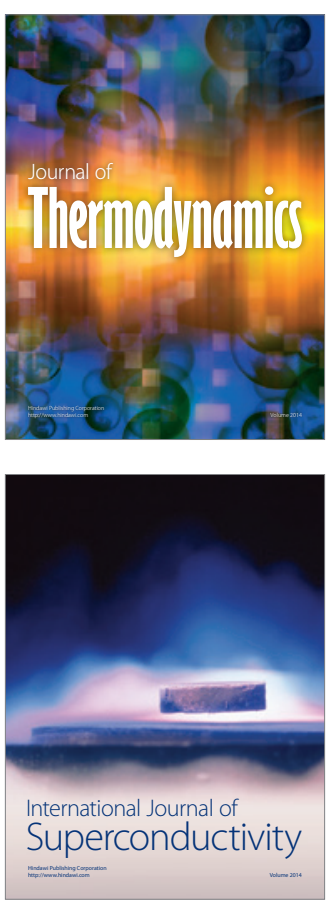
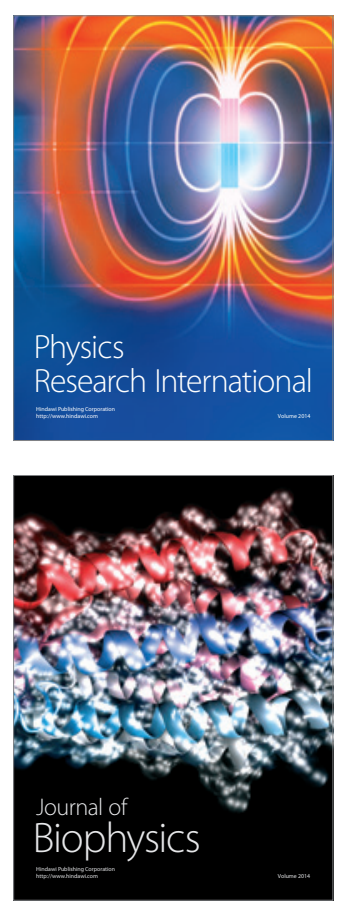
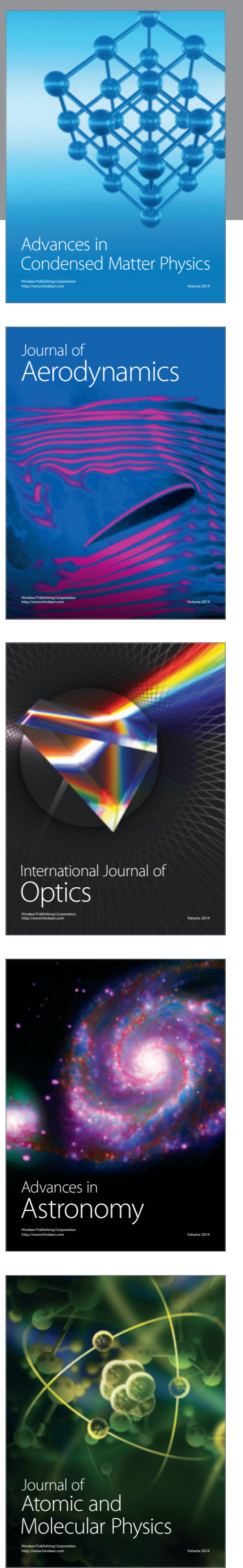